\title{
Some thoughts from the new editors
}

\author{
Lorenzo M. Donini ${ }^{1}$. Giovanni Abbate-Daga ${ }^{2}$
}

Published online: 10 June 2020

(c) Springer Nature Switzerland AG 2020

It is my pleasure and honour to serve as the next editor-inchief of Eating and Weight Disorders (EaWD) journal, along with Prof Giovanni Abbate-Daga as co-editor. On assuming the management of the journal, we would like to offer our great thanks to Prof Massimo Cuzzolaro for his commitment and hard work and acknowledge the significant progress made by EaWD since its foundation.

Under the guidance of Massimo, who founded EaWD 25 years ago, the journal has cut a prestigious space in the field of studies relating to feeding and eating disorders and weight disturbances. This is evidenced by a large number of papers it receives daily, the number of sections and topical collections, and its impact factor which has been progressively increasing.

For clinicians and researchers, Massimo has been an outstanding mentor for all these years and he has helped many colleagues to improve their scientific skills. Working with him for a long time, we appreciated his high competence, his precision, his open-mindedness and his generosity. It helps us to know that we will always have a friend ready to give us good advice for our journal management.

The challenge we face is difficult, but we hope to be able to further improve the journal's international positioning and prestige. This with the help of the section editors who have contributed to better draft the editorial line of EaWD, of the strong international editorial board, of the support of the scientific societies who have endorsed our journal, of the reviewers who have contributed to improving the quality of the published articles, of the authors who show an increasing interest in the journal and, last but not least, of the publisher who has always supported the growth of EaWD.

Our ambition is to improve EaWD reputation also outside of its traditional contribution areas and to encompass all the emerging areas related to eating disorders and obesity while representing the link between scientific research and

\footnotetext{
Lorenzo M. Donini

lorenzomaria.donini@uniroma1.it

Sapienza University, Rome, Italy

2 University of Turin, Turin, Italy
}

professional practice. The challenges in this field are innumerable linked to the new appearance/definition of eating disorders, to the prevalence of these disorders, to face with the advances in medicine and neuroscience, to the increasingly evident consequences that these pathologies have on the clinical, psychological, functional and quality of life level. We need to be able to better understand the pathophysiological bases and clinical manifestations, to validate diagnostic tools, to develop effective therapeutic-rehabilitative pathways, to reflect on the meaning of therapeutic alliance, to involve policymakers in taking care of patients affected by these pathologies. The role of EaWD journal may be fundamental and we wish to be an important player in this way.

The goal of increasing EaWD's ranking will require us to work hard to encourage potential authors to contribute to the journal, to improve the quality of papers, to reduce the time needed for revision, to provide significant reviews and to facilitate the editorial management process.

Finally, we are looking forward to working with you all closely to enhance the reputation of the EaWD journal and we'll be happy to receive your suggestions and ideas for making EaWD more valuable for our research community.

Author contributions The authors contributed equally to the manuscript.

Funding The authors received no specific funding for this work.

\section{Compliance with ethical standards}

Conflict of interest The authors declare that they have no conflict of interest or competing interests.

Ethical approval The article is an Editorial. It did not need ethical approval.

Consent for publication The authors agree to publication.

Publisher's Note Springer Nature remains neutral with regard to jurisdictional claims in published maps and institutional affiliations. 